\title{
Plant Developmental Stage Drives the \\ Differentiation in Ecological Role of Plant Bacterial and Fungal Microbiomes
}

Chao Xiong

Research Centre for Eco-Environmental Sciences Chinese Academy of Sciences

Brajesh K. Singh

Western Sydney University

Ji-Zheng He

The University of Melbourne

Yan-Lai Han

Henan Agricultural University

Pei-Pei Li

Henan Agricultural University

Li-Hua Wan

Soil and Fertilizer Station of Qilin District, Yunnan Province

\section{Guo-Zhong Meng}

Soil and Fertilizer Station of Qilin District, Yunnan Province

Si-Yi Liu

Research Centre for Eco-Environmental Sciences Chinese Academy of Sciences Jun-Tao Wang

Research Centre for Eco-Environmental Sciences Chinese Academy of Sciences

Chuan-Fa Wu

Research Centre for Eco-Environmental Sciences Chinese Academy of Sciences

An-Hui Ge

Research Centre for Eco-Environmental Sciences Chinese Academy of Sciences

Limei Zhang ( $\square$ zhanglm@rcees.ac.cn )

Research Center for Eco-environmental Sciences, Chinese Academy of Sciences https://orcid.org/0000-0002-7383-8475

\section{Research}

Keywords: Crop microbiomes, Temporal dynamics, Soil-plant continuum, Microbiome assembly, Microbial interkingdom networks, Phylloplane microbiome, Metagenomics 
Posted Date: February 25th, 2021

DOl: https://doi.org/10.21203/rs.3.rs-241147/v1

License: (9) This work is licensed under a Creative Commons Attribution 4.0 International License. Read Full License

Version of Record: A version of this preprint was published at Microbiome on August 13th, 2021. See the published version at https://doi.org/10.1186/s40168-021-01118-6. 


\section{Abstract}

\section{Background}

Plants live with diverse microbial communities which profoundly affect multiple facets of host performance such as nutrition acquisition, disease suppression and productivity, but if and how host development impacts the assembly, functions and microbial interactions of crop microbiomes are poorly understood. Here we examined both bacterial and fungal communities across soils (rhizosphere and bulk soil), plant epiphytic and endophytic niches (phylloplane, rhizoplane, leaf and root endosphere), and plastic leaf of fake plant (representing environment-originating microbes) at three developmental stages of maize at two contrasting sites, and further explored the potential function of phylloplane microbiomes based on metagenomics.

Results

Our results suggested that plant developmental stage had a much stronger influence on the microbial diversity, composition and interkingdom networks in plant compartment niches than in soils, with the strongest effect in the phylloplane. Air (represented by fake plants) was an important source of phylloplane microbiomes which were co-shaped by both plant development and seasonal environmental factors. Further, we demonstrated that bacterial and fungal communities in plant compartment niches exhibited contrasting response to host developmental stages, with higher alpha diversity and stronger deterministic assembly within bacterial microbiomes at the early stage but a similar pattern within mycobiomes at the late stage. Moreover, we found that bacterial taxa played a more important role in microbial interkingdom network and crop yield prediction at the early stage, while fungal taxa did so at the late stage. Metagenomic analyses further indicated that phylloplane microbiomes possessed higher functional diversity and functional genes involved in nutrient provision and disease resistance at the early stage than the late stage.

\section{Conclusions}

Our results suggest that host developmental stage profoundly influences plant microbiome assembly and functions, and the bacterial and fungal microbiomes take a differentiated ecological role at different plant development stages. This study provides empirical evidence for host exerting strong effect on plant microbiomes by deterministic selection to meet the physiological requirement of plant developmental stages. These findings have implications for the development of future tools to manipulate microbiome for sustainable increase in primary productivity.

\section{Background}

Plants live with large and diverse prokaryotes and eukaryotes (i.e. plant microbiomes) which have coevolved with their hosts and profoundly impact a range of aspects of plant performance [1-4]. For example, some beneficial bacteria and fungi like nitrogen fixing, antagonistic bacteria and mycorrhizal 
fungi in the rhizosphere and plant compartments, can deeply influence plant growth and health via promoting nutrient acquisition, protecting against pathogen attacks, and increasing tolerance to environmental stress [5-8]. Recent studies suggested that plant microbiome assembly and host health are largely influenced by complex and dynamic interactions between the host, microbes, and the environment, but the ecological processes that govern plant-microbiome-environment interactions remain poorly understood $[3,9]$. A better understanding of the mechanisms and temporal dynamics of plant microbiome assembly, functions and co-occurrence networks is of significant importance for the development of microbiome-based solutions for sustainable crop production systems [10-12].

Assembly of plant microbiomes starts soon after sowing and develops with plant growth under the influence of deterministic (e.g. selection mediated by biotic and abiotic factors) and stochastic (e.g. random dispersal and drift events) processes $[2,8,13]$. It has been suggested that diverse microbial taxa can colonize different plant compartment niches through dispersal from soil, seed and air initially, and then form a dynamic community under the integrative effects of multiple host and environmental factors $[4,8,14-16]$. On the one hand, the plant host has strong selection effects on its microbiomes via host immune system, genetic networks and plant exudates that vary among different compartment niches and host species [13, 17-20]. Further, multiple environmental factors such as climate, edaphic properties (e.g. soil pH and nutrients) and human perturbations (e.g. agricultural management regimes), also play important roles in driving plant microbiome assembly [21-25]. It has been reported that plant microbiomes were mainly determined by compartment niche and host species at the plant level, with the phylloplane and rhizoplane acting as an important interface between the host and the environment [2630]. Some recent studies also highlighted the significant contribution of plant developmental stages on plant microbiome assembly [31-33]. For example, it was suggested that rice root microbiota varied dramatically during the vegetative stages but stabilized after reproduction [33]. This is expected given that plant physiological requirement and composition of plant exudates vary with its developmental stages $[9,13,34,35]$. However, we lack empirical evidence for underpinning the mechanisms of microbiome assembly across soil, plant epiphytic and endophytic niches along with plant developmental stage in field under the interactive influence of climate, edaphic factors and fertilization regimes.

In addition to host and environmental factors, the assembly and stability of the plant microbiome were also strongly affected by microbe-microbe interactions $[2,36,37]$. Potential microbial interactions across different habitats can be characterized using microbial co-occurrence network analysis [37-39]. Previous studies have reported that the network hubs (hub taxa) which frequently interact with other taxa in microbial co-occurrence networks, may act as mediators and gatekeepers for microbial communities and play a crucial role in plant microbiome development, host nutrient acquirement and fitness [13, 37, 40, 41]. Accordingly, a recent study on Arabidopsis root-microbiota showed that microbial interkingdom interactions among bacteria, fungi and oomycetes, could greatly promote Arabidopsis survival in which bacteria played a vital role in protecting plants against pathogens and maintaining microbial interkingdom balance for plant health [41]. The latter suggests the microbial interactions are closely associated with plant fitness. Knowledge is lacking however on bacterial-fungal interactions along the 
soil-plant continuum and how these respond to changes in plant developmental stages, and to what extent these complex interkingdom interactions affect the microbiome dynamics and host performance.

In this study, maize grown in two main agricultural production areas with contrasting soil type and climate condition in China, and received different fertilization practices was used as a study model. The dynamics of both bacterial and fungal communities were investigated over three developmental stages (seedling, tasseling and mature stages) across 432 samples from soils (bulk soil and rhizosphere), multiple plant compartment niches (rhizoplane, root endosphere, phylloplane, leaf endosphere, and grain), and plastic leaf (represented local climate and air environment). Specifically, our aims were to (1) uncover the interactive effect of plant development and environmental factors such as soil type, climate and fertilization practice on microbiome assembly along the soil-plant continuum; (2) identify the interkingdom network patterns and ecological role of bacterial and fungal communities across plant developmental stages. We hypothesized that (1) plant development would dominate over environmental factors in shaping plant microbiomes and vice reverse for soil microbiome, and the phylloplane microbiome would be more dynamic as a result of dual effect from host selection and seasonal environmental factors; (2) The microbial interkingdom network patterns and plant microbiome functions would alter as per the physiological requirement of plant developmental stage, and bacteria and fungi may alternatively function at different stages.

\section{Materials And Methods}

\section{Field experiment description and sampling}

The field experiments were located in Xuchang, Henan province (XC, $34^{\circ} 08^{\prime} 20.4^{\prime \prime} \mathrm{N}, 113^{\circ} 48^{\prime} 34.9^{\prime \prime} \mathrm{E}$;

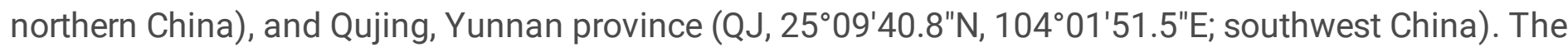
fertilization trial was established in spring of 2016 with seven different fertilization treatments in both study sites: (1) Control (zero nitrogen fertilizer); (2) N (local regime' N rate); (3) $80 \% \mathrm{~N}$ ( $20 \% \mathrm{~N}$ reduction based on $\mathrm{N}$ treatment); (4) $80 \% \mathrm{NS}(80 \% \mathrm{~N}$ treatment plus straw covering); (5) $80 \% \mathrm{NI}(80 \% \mathrm{~N}$ treatment plus nitrification inhibitor chlorinated pyridine applied); (6) $80 \% \mathrm{NKle}(80 \% \mathrm{~N}$ treatment plus foliar spraying of an nitrogen-fixing bacteria Klebsiella variicola W12); (7) 80\%NSB (80\%NS treatment plus biochar addition). The two field experiments were random block designed with three replicate plots (about $30 \mathrm{~m}^{2}$ for each) and managed according to local agriculture practices. To estimate the influence of seasonal environmental factors (e.g. air, dust, temperature, rainfall and UV etc.) on maize phylloplane microbiome, fake plants made of plastic material with a height of $1.5 \mathrm{~m}$ were planted as "background controls" in the field when the maize was sown.

Sample collections in two sites were performed at June, August and September of 2017, corresponding to maize seedling, tasseling and mature stage, respectively. For each time point, leaf, root, rhizosphere soil, and bulk soil samples were collected from each plot following our previous method $[29,42]$. The plastic leaf samples were also collected from each time point and maize grain samples were collected at the mature stage. As the study mainly aimed at the temporal dynamics of soil and crop microbiomes, we 
focused on six compartments (bulk soil, rhizosphere, rhizoplane, root endosphere, phylloplane and leaf endosphere) in three treatments (Control, $80 \% \mathrm{~N}$, and $80 \% \mathrm{NS}$ ). We also included phylloplane samples from other four treatments $(\mathrm{N}, 80 \% \mathrm{NI}, 80 \% \mathrm{NKle}, 80 \% \mathrm{NSB})$, as our previous studies suggested that the phylloplane niche was a hotspot of plant-microbes-environment interactions and affected by both host and environments [29]. In total, we collected 432 samples for microbial community analysis. More information on fertilization treatment, field management and sampling is provided in supplementary materials "Method S1" and our previous publications [29, 42].

All samples for molecular work were transported to the lab on dry ice and stored at $-80^{\circ} \mathrm{C}$ until further processing. Soil physicochemical characteristics (e.g. $\mathrm{pH}_{1} \mathrm{NH}_{4}{ }^{+}-\mathrm{N}$ and $\mathrm{NO}_{3}{ }^{-} \mathrm{N}$ ) and enzyme activities related with $\mathrm{C}, \mathrm{N}$, and $\mathrm{P}$ cycling (e.g. nitrogenase activity and phosphatase) were measured according to previous protocols [42-44].

\section{DNA extraction}

The rhizosphere and bulk soil DNA were extracted from $0.4 \mathrm{~g}$ soil using the PowerSoil DNA Isolation Kit (MO BIO Laboratories, Carlsbad, CA, USA) according to manufacturer's instructions. The epiphytic and endophytic microbial cells from the leaves (10-15 g) and roots (3-5 g) were collected following our previous method [29], and subjected to DNA extraction using the PowerSoil DNA Isolation Kit. The epiphytic DNA from the plastic leaves $(10-15 \mathrm{~g})$ was obtained using the same method for the maize leaf epiphytic DNA collection. For maize grain, $\sim 5 \mathrm{~g}$ sample was ground using sterile mortars and pestles with liquid nitrogen and then DNA was extracted from the $0.4 \mathrm{~g}$ resulting powder using the PowerSoil DNA Isolation Kit.

\section{Amplicon sequencing and bioinformatic analysis}

Bacterial 16S rRNA gene V5-V6 region was amplified using primers 799F and 1115R [45], and fungal ITS2 region was amplified using primers fITS7 [46] and ITS4 [47]. Sequencing was performed on the Illumina MiSeq platform with a Paired-End protocol. The raw sequences were quality-filtered using USEARCH (v10.0) [48] as previously described [29, 42], and all correct biological reads (i.e. zero-radius operational taxonomic units, ZOTUs) were picked at $100 \%$ similarity using unoise3 command [49] with default parameters. Bacterial and fungal sequences were classified using SILVA (v13.2) and UNITE (v8.0) databases, respectively. Bacterial ZOTUs assigned to chloroplast, mitochondrial and viridiplantae, and fungal ZOTUs assigned to plant or protist were removed. Both bacterial and fungal ZOTUs represented by less than 2 sequences were removed to avoid possible biases. In total, 11,665,748 bacterial and $23,156,931$ fungal high quality reads from 432 samples were retrieved and sorted into 18,602 bacterial and 9,299 fungal ZOTUs (i.e. phylotypes; analogous to amplicon sequence variants). Both bacterial and fungal alpha- and beta-diversity were calculated in QIIME [50]. Bacterial and fungal ZOTU tables were then rarefied to 3130 and 33000 reads for the alpha diversity estimates, respectively. For beta diversity analyses, ZOTU tables were normalized using the cumulative-sum scaling (CSS) method [51]. 


\section{Metagenomic sequencing and data mining}

To further characterize phylloplane microbiome functions, we selected 9 maize phylloplane (based on the $\mathrm{N}$ treatment, 3 replicates $\times 3$ stages) and 9 plastic leaf (3 replicates $\times 3$ stages) DNA samples from the site QJ for metagenomic sequencing using the Illumina NovaSeq platform with a Paired-End protocol. Raw sequences were quality-filtered using Trimmomatic (v0.39) [52], and sequences belonging to the maize genome were removed by mapping the data to the maize reference genome (NCBI: NC_050096.1) with Bowtie2 (v2.1.0) [53]. Finally, an average of $8.6 \mathrm{~Gb}$ of clean data was retrieved for each sample. These high-quality reads were assembled using Megahit (v1.2.9) [54], and then were predicted using Prokka (v1.14.5) [55] and clustered with a 0.95 similarity threshold using CD-HIT (v4.8.1) to generate nonredundant gene catalog. The functional profiles (including KO, CAZy and COG) of phylloplane microbiome were determined using eggNOG databases (v5.0) [56], and rarefied based on the lowest reads among all samples. The Chao1 index of functional diversity was calculated in QIIME.

\section{Statistical analysis}

The linear mixed model analysis was performed to identify the major drivers of microbial alphadiversity [57]. The beta-diversity of both bacterial and fungal communities was assessed by computing weighted UniFrac distances matrices and then ordinated using non-metric multi-dimensional scaling (NMDS). The relative contribution of different biotic and abiotic factors on community dissimilarity was tested with PERMANOVA [58]. To assess the relative importance of determinism and stochasticity in microbiome assembly, we calculated the beta Nearest Taxon Index ( $\beta N T I)$ using null model (999 randomizations) [59], and defined $\mid \beta N T \| \geq 2$ as dominant deterministic processes and $|\beta N T| \mid<2$ as dominant stochastic processes $[60,61]$. Further, deterministic and stochastic processes were partitioned into five ecological processes based on both $\beta N T I$ and Bray-Curtis-based Raup-Crick Index $\left(\mathrm{RC}_{\text {Bray }}\right)$ values, including heterogeneous selection ( $\beta N T I<-2)$, homogeneous selection $(\beta N T I>+2)$, dispersal limitation $\left(|\beta N T| \mid<2\right.$ and $\left.R C_{\text {Bray }}>0.95\right)$, homogenizing dispersal $\left(|\beta N T| \mid<2\right.$ and $\left.\mathrm{RC}_{\text {Bray }}<-0.95\right)$, and undominated $\left(|\beta N T| \mid<2\right.$ and $\left.\left|\mathrm{RC}_{\text {Bray }}\right|<0.95\right)[60,61]$.

Microbial interkingdom network analysis at bacterial and fungal genus level was performed using the CoNet [62] in Cytoscape (v3.5) [63] based on Spearman correlation scores (Spearman's $r>0.7$ or $r<-0.7$; $P<0.01)$. Both bacterial and fungal genera present in at least 10 samples were retained for the network analysis [42]. The networks were visualized in Gephi [64]. Bacterial functional profiles were predicted using functional annotation of prokaryotic taxa (FAPROTAX) [65], and fungal functional groups were predicted using the program FUNGuild [66]. The Source Model of Plant Microbiome (SMPM) was estimated using SourceTracker (v1.0) [67]. Differential abundance analysis was performed using EdgeR's generalized linear model (GLM) approach [68]. Random forest analysis was conducted using the "randomForest" R package [69]. Mantel test was performed to explore the correlations between microbial communities, soil physicochemical characteristics, and soil enzyme activities using the "vegan" package [42]. All statistical analyses were carried out in R (http://www.r-project.org). Nonparametric 
statistical test (Kruskal-Wallis test or Wilcoxon test) was performed to evaluate the alpha-diversity difference and the taxonomical difference among different niches and stages. More information on the network analysis, random forest modeling analysis and source tracking analysis are detailed in our previous publication $[29,42]$.

\section{Results}

\section{Diversity and community assembly of bacterial and fungal microbiomes across three plant developmental stages}

The linear mixed model analysis suggested that plant developmental stages had a greater influence on both bacterial and fungal Chao1 richness in plant compartment niches (phylloplane, leaf endosphere, rhizoplane and root endosphere) than those in the rhizosphere and bulk soils (Table S1). For plant compartment niches, bacterial Chao1 richness decreased from the seedling stage to the mature stage, while fungi showed opposite pattern (Fig. 1a and Fig. S1a, b).

PERMANOVA analysis and NMDS ordinations indicated that plant developmental stage explained the largest variations (16-45\%) in both bacterial and fungal communities in most plant compartment niches (phylloplane, leaf endosphere and root endosphere), with the strongest effect in the phylloplane (bacteria: 45.1\%; fungi: 39.6\%) (Fig. 1c; Table S2). In contrast, microbial communities in the rhizoplane, rhizosphere, and bulk soil were primarily explained by site (31-65\%) (Fig. 1c; Table S2). Regression analysis based on Bray-Curtis dissimilarity also showed similar patterns where plant developmental stage had the strongest effect on microbial communities in the phylloplane (Fig. S2). At the plant level, microbiome assembly was mainly explained by compartment niche (bacteria: $R^{2}=54.8 \%$; fungi: $R^{2}=39.6 \%$ ), followed by developmental stage (bacteria: $R^{2}=6.1 \%$; fungi: $R^{2}=5.4 \%$ ) and site (bacteria: $R^{2}=3.7 \%$; fungi: $R^{2}=$ $7.5 \%$ ) (Fig. 1b; Table S2). Fertilization practice played a marginal role in driving microbial communities for all samples and samples within each niche (Table S2). Moreover, microbial community dissimilarity among all samples was much lower at the seedling stage than at other stages (Fig. S1a, b). Although maize phylloplane microbiomes significantly differed from plastic leaf microbiomes $(P<0.01)$, both of them showed similar temporal variation patterns (Fig. 1b, c; Table S2).

Null model analysis showed that the relative contribution of deterministic $(|\beta N T| \mid \geq 2)$ and stochastic ( $\beta N T I<2$ ) processes in crop microbiome assembly were greatly affected by plant developmental stage, particularly for the phylloplane and leaf endosphere (Fig. 2a, b). At the seedling stage, a higher relative contribution of deterministic processes mainly belonging to heterogeneous selection in plant compartments was observed in bacterial communities ( $71 \%)$ than in fungal communities $(\sim 47 \%)$. Conversely, the effects of deterministic processes decreased for bacterial communities (to $~ 53 \%$ ) but increased for fungal communities (to $\sim 64 \%$ ) at the mature stage (Fig. 2a, b). Collectively, deterministic processes exerted a greater influence on crop bacterial microbiome at the early stage and on mycobiome at the late stage, respectively. By contrast, microbial communities in the rhizosphere (71-81\%), bulk soil 
(61-76\%) and plastic leaf (87-100\%) were mainly driven by deterministic processes over the time (Fig. 2a, b).

\section{Temporal dynamics of microbial interkingdom co-occurrence networks}

We further performed co-occurrence network analysis to assess the impact of host developmental stage on bacterial-fungal interkingdom interactions along the plant-soil continuum. Our results showed that microbial interkingdom network patterns shifted clearly across three developmental stages, with differentiated bacterial and fungal roles in the networks during plant development (Fig. 3a-e). Specifically, bacterial taxa had higher network connectivity (i.e. network degree) than fungal taxa at the seedling stage, while the pattern was reverse at the mature stage (Fig. 3a-d). In contrast, fungal network connectivity increased from 2.2 at the seedling stage to 17.8 at the mature stage (Fig. 3a-d). Moreover, the proportion of negative network edges (mainly representing bacteria-fungi interkingdom correlations) markedly increased from $6.1 \%$ at the seedling stage to $50.5 \%$ at the mature stage (Fig. 3a-C, e). We further defined the "network hubs" as node with high values of degree $(>50)$ and closeness centrality $(>0.3)$ in the network, and found 3 network hubs (bacteria: 3 ; fungi: 0 ) at the seeding stage, and 2 (bacteria: 1; fungi: 1) at the tasseling stage, and 10 (bacteria: 4; fungi: 6) at the mature stage (Fig. 3d; Table S3). Similar patterns were also recorded in microbial interkingdom functional networks based on function prediction of amplicon sequencing data, with more bacterial functional network hubs (e.g. group "nitrate respiration") at the first two stages (Fig. S3a-e). In contrast, the highest number of fungal functional network hubs was identified at the mature stage and mainly represented by group "Saprotroph" (Fig. S3ad).

As for each niche, microbial interkingdom network patterns in plant compartments changed distinctly across three developmental stages, particularly for the phylloplane and leaf endosphere (Fig. S4a-c). The fungal network connectivity and the proportion of fungal nodes significantly increased from the seedling stage to the mature stage in all four plant compartmens (Fig. S4a-c). Conversely, the network patterns in the rhizosphere and bulk soil were relatively stable over the three stages (Fig. S4a-c).

\section{Temporal dynamics of microbiome composition across soil, plant epiphytic and endophytic niches}

Taxonomic classification showed that both bacterial and fungal communities in plant compartment niches varied distinctly across three developmental stages, but not in the rhizosphere and bulk soils (Fig. 4a). The linear mixed model analysis based on the dominant bacterial and fungal phyla/classes (top 10) suggested that plant developmental stage had the strongest effects on bacterial phyla Actinobacteria ( $F$ $=132.7, P<2.2 \mathrm{e}-16)$ and Bacteroidetes $(F=78.4, P<2.2 \mathrm{e}-16)$, and fungal classes Sordariomycetes $(F=$ 34.0, $p=8.3 \mathrm{e}-14)$ and Dothideomycetes $(F=20.0, p=1.6 \mathrm{e}-8)$. Notably, we found that Actinobacteria in plant compartments was more abundant at the seedling stage $(26.1 \%)$ than at the tasseling stage 
$(15.9 \%)$ and the mature stage $(12.1 \%, P<0.001)$ (Fig. 4a, Fig. S5a). In addition, the relative abundance of Dothideomycetes in the rhizoplane increased from the seedling stage to the mature stage $(P<0.001)$, while the Dothideomycetes in the root endosphere showed an opposite pattern (Fig. 4a, Fig. S5a). Notable, Dothideomycetes dominated mycobiomes in the plastic leaf and maize phylloplane in both sites and showed no visible variation among three developmental stages (Fig. 4a). Differential abundance analysis at ZOTU level further showed that some members within bacterial families Burkholderiaceae, Microbacteriaceae, Streptomycetaceae and Rhizobiaceae were significantly enriched in at least two plant compartments (e.g. phylloplane and rhizoplane) at the seedling stage (Fig. S6a, b; Table S4). Moreover, some members within fungal families Coniothyriaceae, Mycosphaerellaceae, Sporidiobolaceae and Symmetrosporaceae were significantly enriched in at least two plant compartments at the mature stage (Fig. S6a, b; Table S4), and some genera within these families were identified as hubs in microbial interkingdom network at the mature stage (Fig. 3a-d; Table S3).

Furthermore, the random forest modeling analysis indicated that bacterial community composition at the seedling stage is a strong predictor for crop yield while fungal community composition at the mature stage did so (Fig. 4b). Mantel test showed that bacterial communities of the rhizosphere and bulk soils in both sites had significant correlations with soil $\mathrm{N}$ cycling related enzyme activities like nitrogenase and potential nitrification rate across three developmental stages. In contrast, fungal communities had significant correlations with soil enzyme activities related with $C$ and $P$ cycling like $\beta$-glucosidase and phosphatase (Fig. S7a-b; Table S5).

The Source Model of Plant Microbiome (SMPM) showed that maize-associated bacterial and fungal communities were mainly derived from bulk soils and gradually filtered at different plant compartment niches, and the trends were similar across three developmental stages (Fig. S8a; Table S6). Maize grain potentially selected majority of taxa from leaves (bacteria: $46.5 \%$, fungi: $28.4 \%$ ) and roots (bacteria: $39.6 \%$, fungi: $58.1 \%$ ) (Fig. S8a). Remarkably, environment-originated (represented by the plastic leaf) microbiomes were important sources of maize phylloplane microbiomes, with an increasing contribution from $52.6 \%$ to $87.2 \%$ for bacteria and from $86.6 \%$ to $92.4 \%$ for fungi across three stages (Fig. S $8 b$ ).

\section{The functional profiles of phylloplane microbiomes}

Metagenomic analysis indicated that the functional composition (i.e. NMDS ordinations of KEGG Orthology) of maize phylloplane microbiome significantly differed from that of plastic leaf of fake plant $\left(R^{2}=44.3 \%, P<0.01\right)$, and the developmental stage also had significant effect on phylloplane microbiome functions for both maize $\left(R^{2}=50.0 \%, P<0.01\right)$ and fake plant $\left(R^{2}=84.1 \%, P<0.01\right)$ (Fig. $5 a)$. Importantly, the maize phylloplane possessed higher microbiome functional diversity (i.e. Chao1 richness based on KO, CAZy and COG) at the seedling stage than at other stages, while the plastic leaf showed opposite pattern (Fig. 5a). Differential abundance analysis showed that the numbers of specifically enriched functional traits (including KO, CAZy and $\mathrm{COG}$ ) in maize phylloplane was significantly higher at the seedling stage than at any other stages, with functional gene involved in 
disease resistance (K13457) significantly enriched at the seedling stage (Fig. 5b). Moreover, functional genes involved in nitrogen fixation (e.g. nifH, nifD, nifK) and nitrate reduction (e.g. nrfA and napA) in maize phylloplane were more abundant at the seedling stage, while nitrous oxide reductase gene (nosZ), $\mathrm{N}$ assimilation gene (nas $A$, nas $B$ ) and $\mathrm{C}$ degradation (e.g. $x y / A$ and amy $A$ ) and $\mathrm{P}$ transport (e.g. pst $A$ and pstB) related genes were more abundant at the other two stage (Fig. $5 \mathrm{c}$ ). Some functional attributes related with methyl-accepting chemotaxis (K03436), inorganic ion transport (COG_P) and defense mechanisms (COG_V) were identified as the biomarker functions for the maize phylloplane while chitin synthase (K00698) was identified for fake plant by LEfSe (Fig. S9a). Although both maize and fake plant shared $39-55 \%$ of phylloplane ZOTUs across three stages (Fig. S9b), a stronger depleted effect was observed in the maize phylloplane when compared with fake plant. Some ZOTUs mainly belonging to Alphaproteobacteria and Actinobacteria, like ZOTU7 (Microbacterium) and ZOTU9985 (Sphingomonas), were significantly enriched in the maize phylloplane over three stages (Fig. S9b).

\section{Discussion}

\section{Plant development strongly influences the assembly of plant microbiomes}

Uncovering the ecological principles and processes that underpin plant microbiome assembly and developmental dynamics is essential to advance fundamental understanding of co-evolution and future application of the crop microbiome to sustainable increase in farm productivity $[9,10,70]$. Our results demonstrate that maize microbiome assembly is mainly influenced by compartment niche and developmental stage regardless of farming regions and fertilization regimes. Further, plant microbiomes are more sensitive to plant developmental stages than soil microbiomes in terms of multiple microbial attributes (i.e. alpha diversity, community structure, assembly processes and interkingdom networks). These findings are consistent with previous studies showing that plant compartment is a determining factor shaping the assembly of plant-associated microbiomes [26-29, 42], and that plant seasonal status has significant effects on microbiomes in grasses phyllosphere and Arabidopsis rhizosphere [32, 71]. Further, metagenomic analysis in our study revealed that the functional diversity and enriched functional traits in the maize phylloplane varied across three stages. There was a stronger depletion effect in the maize phylloplane in comparison to fake plant phylloplane, and functional attributes involved in methylaccepting chemotaxis and defense mechanisms were significantly enriched in the maize phylloplane. Together these results indicate that the plant host exerts a strong selection effect to recruit and filter specific microbial taxa and functions from nearby species pool during plant development $[18,72,73]$. Complementary to the previous finding that host selection via plant compartment niche and host genetics plays a dominant role in shaping plant microbiomes assembly [20, 26, 29, 31, 42], this work provides novel evidence that plant developmental stages profoundly influence not only plant microbiome assembly but also their functions.

The effects of plant developmental stage represented the dynamic effects of plant metabolism, exudation and immune-associated traits $[9,13,74]$, and plant-associated microbes have strong 
chemotaxis activities towards plant signal molecules such as organic acids and sugars [34, 71, 75-77]. For instance, a recent work revealed that wheat root-released organic carbon varied dramatically across wheat growth stages and correlated with different microbial taxa [34]. It was also suggested that plant exudates and volatiles like coumarins, benzoxazinoids and triterpenes play key roles in shaping plant microbiomes during host development $[17,72,76]$. The effect of plant developmental stages on microbiome in this study included the effects from season-dependent environmental factors like air, dust and climate. Our results showed that both maize and fake plant phylloplane microbiomes had similar temporal patterns and shared more than one third of ZOTUs at each stage. Further, fake plant phylloplane microbiome contributed an increasing proportion as the source of the maize phylloplane microbiome over the time, indicating a part contribution of environmental temporal factors to crop microbiome assembly. These results presented strong field evidence showing that local air, dust and rainwater are the main sources of crop microbiome in phyllosphere. These findings significantly advance our knowledge on the source, driving force and potential function of phyllosphere microbiome, and further corroborated that the phylloplane acts as an important interface between the host, microbes, and the environment [30, 78-80]. Our results also showed that plant developmental stage had significant effects on the rhizosphere and bulk soil microbiomes, though it was much weaker than the site effects, implying that plants also have profound influence on soil microbiomes via strong rhizosphere effect $[6,35,74]$. Collectively, by examining the temporal dynamics of bacterial and fungal microbiomes in the soil-plant continuum of maize and fake plants phylloplane in geographically distant sites, this study considerably expanded our knowledge on the succession of plant microbiomes and their potential function under different temporal and spatial scales in field.

\section{The differentiation in ecological roles of bacterial and fungal microbiomes across plant developmental stages}

Bacteria and fungi have coevolved with their host for more than 400 million years and greatly contribute to numerous aspects of plant health and productivity $[1,8,41]$. In this study, bacterial-fungal interkingdom interaction patterns distinctly shifted across three developmental stages. Bacterial community possessed higher alpha diversity and network connectivity at the seedling stage while fungal diversity was higher at the mature stage. Moreover, bacterial and fungal taxa dominated network hubs at the seedling stage and the mature stage, respectively. These suggested that the host can selectively modulate microbial interactions to meet its requirement during plant development, as microbial network hubs were supposed to play crucial roles in maintaining plant health and nutrient [37, 40]. In addition, bacterial taxa at the seedling stage were better predictors of crop yield while fungal taxa at the mature stage did so. Metagenomic analysis further corroborated that maize phylloplane microbiome possessed higher functional diversity at the seedling stage than the other two stages. Importantly, more abundant genes associated with disease resistance were enriched at the seedling stage while $C$ degradation and $P$ transportation related genes were enriched at two late stages, which further support the ability of plants to manipulate microbial assembly as per physiological requirements $[1,73]$. Based on the limited knowledge on the plant microbiome, it has been proposed that the dynamics of plant microbiome 
composition are a reflection of the current needs of the host plant $[3,40,73]$, and represent the consequence of subtle changes in microbial selection strategy exerted by the host during plant development $[1,13,73,81]$. Our results therefore support that bacteria may take a more important ecological role in the plant microbiome and host performance at the early stage, while fungi do so at the late stage. This finding is supported by the Null model analysis, which demonstrated the dominant effect of determinism on bacterial community and of stochasticity on fungal community at the seedling stage, but a reverse pattern at the mature stage.

We further found that the negative edges representing bacterial-fungal interkingdom correlations in network increased over the time, implying an increasing competition relationship between bacteria and fungi along plant development stages. It was suggested that microbial competitive interaction could positively influence microbiome stability $[40,82,83]$. Our study provides more empirical evidence on this and further support the argument that the host may facilitate host fitness and plant-microbiome balance by deterministic host selection during plant development. These findings provide new insights into complex interactions among the plant, microbes and the environment and provide essential information for the future development of tools to manipulate crop microbiome.

\section{Keystone bacterial and fungal taxa and their ecological functions at different developmental stages}

Our results suggest that the composition and potential functions of plant microbiomes change across plant growth and bacterial phyla Actinobacteria and Bacteroidetes were more sensitive in response to plant developmental stage. More abundant Actinobacteria were observed at the seedling stage than at two late stages in plant compartments. Actinobacteria are well known as antagonistic bacteria excreting antibiotic compounds that provide protection against plant pathogens [84-86]. For example, a recent work showed that the enrichment of protective microbes like Actinobacteria in the rhizosphere could facilitate disease suppression [85]. Furthermore, some ZOTUs within families Burkholderiaceae, Streptomycetaceae and Rhizobiaceae were significantly enriched in plant compartment niches at the seedling stage. The members within Burkholderiaceae and Rhizobiaceae are important diazotrophs and plant growth promoting bacteria (PGPR) $[1,6,12]$, and the members within Streptomycetaceae are wellknown antibiotic-producing bacteria $[41,87,88]$. Coincidently, metagenomic analysis for the maize phylloplane suggested that functional genes involved in disease resistance and $\mathrm{N}_{2}$ fixation were significantly enriched at the seedling stage. In addition, bacterial communities in the rhizosphere and bulk soils showed significant correlations with nitrogenase activity across three developmental stages, and the bacterial functional group "nitrite respiration" was identified as the network hubs at the seedling stage. These findings provide evidence for the ecological importance of crop bacterial microbiomes in maintaining plant health and nutrient requirement at the early stage.

For fungal community, classes Sordariomycetes and Dothideomycetes were more sensitive to plant development, with Dothideomycetes increasing over the time in the rhizoplane but decreasing in the root 
endosphere. Previous works have shown that Sordariomycetes and Dothideomycetes are the most dominant fungal taxa in soils and plant compartments, respectively, and that class Dothideomycetes comprises a highly diverse range of fungi including endophytes, epiphytes and plant pathogens $[42,89]$. In addition, many members within Dothideomycetes are also identified as saprotrophic fungi functioning in wood and leaf-litter decomposition and nutrient cycling [89, 90]. Notably, Dothideomycetes predominated phylloplane mycobiomes of both fake plant and maize in two distant study sites across three developmental stages. It was suggested that Dothideomycetes are the dominant fugal taxa of air microbiomes [90]. This indicated that Dothideomycetes in fake plant and maize phylloplanes might be mainly dispersed from air. The reverse dynamics of Dothideomycetes in the rhizoplane and root endosphere indicated multiple ecological roles of Dothideomycetes during host growth, however, its biological functions need further exploration. Furthermore, some fungal ZOTUs affiliating within families Coniothyriaceae, Mycosphaerellaceae and Symmetrosporaceae were identified as network hubs and significantly enriched in plant compartments at the mature stage. Some members of families Coniothyriaceae and Mycosphaerellaceae within Dothideomycetes are important saprobes with celluloseand carbohydrate-degrading ability $[90,91]$. Coincidently, we found that most network hubs in both taxonomic and functional networks of the mature stage belonged to fungal functional group "Saprotroph". Moreover, fungal communities in the rhizosphere and bulk soils had significant correlations with $\mathrm{C}$ cycling related enzymes like $\beta$-glucosidase across three developmental stages. These results suggest that fungal taxa play key roles in regulating plant $\mathrm{C}$ cycles like decomposition of plant residues at the late stage. This indicates that crop mycobiomes may play an increasing ecological role as the decomposers with the aging of the plant, and the host plant may be passively occupied by saprophytic fungi as the consequence of reduced host immunity.

Collectively, our study demonstrates that plant is able to recruit specific microbial taxa with desire functions to meet their health and nutrient requirements at different developmental stages. However, the molecular mechanisms governing plant-microbiome interactions during host development and the ecological and biological functions of crop microbiomes in facing climate challenge and achieving sustainable agriculture are not fully understood and need further exploration $[10,92,93]$.

\section{Conclusions}

By examining the temporal dynamics of bacterial and fungal communities across soils, multiple plant compartments and fake plant phylloplane at two geographically distant sites, this study provides a systematic understanding on the succession of microbiome composition and their potential functions during plant development. Our results demonstrate that plant developmental stage has a much stronger influence on multiple microbial attributes (i.e. alpha diversity, community structure, determinism/stochasticity patterns and interkingdom networks) in plant compartment niches than in soils, with the strongest effect in the phylloplane. We further found that air is an important source of phylloplane microbiomes, which were strongly co-shaped by plant development and seasonal environmental factors. Furthermore, we demonstrate that the ecological role of bacterial and fungal microbiomes significantly shifts with plant development, along which bacteria take a more important role 
in microbial interkingdom networks and crop yield prediction at the early stage while fungi take an increasing role at the late stage. Metagenomic analyses further revealed that maize phylloplane microbiome possessed higher functional diversity and functional genes involved in disease resistance and nutrient provision at the early stage than at the late stage. In contrast, more abundant $\mathrm{N}$ assimilation and $\mathrm{C}$ degradation genes were observed in maize phylloplane microbiomes at the late stage, corresponding to an increasing ecological importance of saprophytic fungi in plant microbiomes. Additionally, we found a dominant effect of determinism on bacterial microbiomes at the early stage and on mycobiomes at the late stage in plant compartments. Together these results suggest that the host has a strong selective modulation effect on their microbiomes which is strongly modified by the plant developmental stages. These findings significantly advance our fundamental understanding of plantmicrobiome interactions and provide critical new knowledge for future synthetic community research, and the development of microbiome tools to enhance plant protection and agriculture production in a sustainable way.

\section{Declarations}

\section{Acknowledgements}

We would like to thank Prof. Yong-Guan Zhu, Dr. Hang-Wei Hu and Dr. Yin Chen for their suggestions and comments on experiment design and manuscript writing, and Mr. Qin-Bing Zhang and Hao-Tian Tong for their cooperation and assistance in field management and sampling. We also thank Mr. Feng Dai for supplying the nitrification inhibitor chlorinated pyridine (CP).

\section{Authors' contributions}

LMZ, JZH, and CX designed the study. LMZ, SYL, GZM, LHW, YLH and PPL managed the field trial stations. CX, CFW and AHG collected samples. CX conducted the laboratory analyses. CX, JTW, BS and LMZ performed the data processes. CX, LMZ, BS, JTW and JZH wrote the manuscript.

\section{Funding}

This work was financially supported by National Key R\&D Program (2017YFD0200600) and the Strategic Priority Research Program (B) of Chinese Academy of Sciences (XDB15020200). Research on plant and soil microbiomes in BKS lab is supported by Australian Research Council (DP170103628; DP190103714). Li-Mei Zhang was supported by the Youth Innovation Promotion Association, Chinese Academy of Sciences (Y201615).

\section{Availability of data and materials}




\section{Ethics approval and consent to participate}

Not applicable.

\section{Consent for publication}

Not applicable.

\section{Competing interests}

The authors declare that they have no competing interests.

\section{Author details}

${ }^{1}$ State Key Laboratory of Urban and Regional Ecology, Research Center for Eco-Environmental Sciences, Chinese Academy of Sciences, Beijing 100085, China. ${ }^{2}$ University of Chinese Academy of Sciences, Beijing 100049, China. ${ }^{3}$ Global Centre for Land-Based Innovation, Western Sydney University, Penrith, NSW 2751, Australia. ${ }^{4}$ Hawkesbury Institute for the Environment, Western Sydney University, Penrith, NSW 2751, Australia. ${ }^{5}$ Faculty of Veterinary and Agricultural Sciences, The University of Melbourne, Parkville, VIC 3010, Australia. ${ }^{6}$ College of Resource and Environmental Sciences, Henan Agricultural University, Zhengzhou 450002, China. ${ }^{7}$ Soil and Fertilizer Station of Qilin District, Qujing, Yunnan Province, Qujing, 655000 , China.

\section{References}

1. Martin FM, Uroz S, Barker DG. Ancestral alliances: plant mutualistic symbioses with fungi and bacteria. Science. 2017;356:eaad4501.

2. Muller DB, Vogel C, Bai Y, Vorholt JA. The plant microbiota: systems-level insights and perspectives. Annu Rev Genet. 2016;50:211-34.

3. Fitzpatrick CR, Salas-González I, Conway JM, Finkel OM, Gilbert S, Russ D, et al. The plant microbiome: from ecology to reductionism and beyond. Annu Rev Microbiol. 2020;74:81-100.

4. Vandenkoornhuyse P, Quaiser A, Duhamel M, Le Van A, Dufresne A. The importance of the microbiome of the plant holobiont. New Phytologist. 2015;206:1196-206.

5. Laforest-Lapointe I, Paquette A, Messier C, Kembel SW. Leaf bacterial diversity mediates plant diversity and ecosystem function relationships. Nature. 2017;546:145-7. 
6. Philippot L, Raaijmakers JM, Lemanceau P, van der Putten WH. Going back to the roots: the microbial ecology of the rhizosphere. Nat Rev Microbiol. 2013;11:789-99.

7. Mendes R, Kruijt M, de Bruijn I, Dekkers E, van der Voort M, Schneider JH, et al. Deciphering the rhizosphere microbiome for disease-suppressive bacteria. Science. 2011;332:1097-100.

8. Hassani MA, Duran P, Hacquard S. Microbial interactions within the plant holobiont. Microbiome. 2018;6:58.

9. Trivedi P, Leach JE, Tringe SG, Sa T, Singh BK. Plant-microbiome interactions: from community assembly to plant health. Nat Rev Microbiol. 2020;18:607-21.

10. Singh BK, Trivedi P, Egidi E, Macdonald CA, Delgado-Baquerizo M. Crop microbiome and sustainable agriculture. Nat Rev Microbiol. 2020;18:601-2.

11. Busby PE, Soman C, Wagner MR, Friesen ML, Kremer J, Bennett A, et al. Research priorities for harnessing plant microbiomes in sustainable agriculture. PLoS Biol. 2017;15:e2001793.

12. Haskett TL, Tkacz A, Poole PS. Engineering rhizobacteria for sustainable agriculture. ISME J. 2020;doi: 10.1038/s41396-020-00835-4.

13. Viviane C, Francisco DA, Víctor JC, Jos MR. Ecology and evolution of plant microbiomes. Annu Rev Microbiol. 2019;73:69-88.

14. Vorholt JA, Vogel C, Carlstrom Cl, Muller DB. Establishing causality: opportunities of synthetic communities for plant microbiome research. Cell Host Microbe. 2017;22:142-55.

15. Bai Y, Mueller DB, Srinivas G, Garrido-Oter R, Potthoff E, Rott M, et al. Functional overlap of the Arabidopsis leaf and root microbiota. Nature. 2015;528:364-9.

16. Bulgarelli D, Schlaeppi K, Spaepen S, Ver Loren van Themaat E, Schulze-Lefert P. Structure and functions of the bacterial microbiota of plants. Annu Rev Plant Biol. 2013;64:807-38.

17. Harbort CJ, Hashimoto M, Inoue H, Niu Y, Guan R, Rombola AD, et al. Root-secreted coumarins and the microbiota interact to improve iron nutrition in Arabidopsis. Cell Host Microbe. 2020;28:82537.e6.

18. Shakir S, Zaidi SS-e-A, de Vries FT, Mansoor S. Plant genetic networks shaping phyllosphere microbial community. Trends Genet. 2020;doi: 10.1016/j.tig.2020.09.010.

19. Walters WA, Jin Z, Youngblut N, Wallace JG, Sutter J, Zhang W, et al. Large-scale replicated field study of maize rhizosphere identifies heritable microbes. Proc Natl Acad Sci U S A. 2018;115:736873.

20. diCenzo GC, Checcucci A, Bazzicalupo M, Mengoni A, Viti C, Dziewit L, et al. Metabolic modelling reveals the specialization of secondary replicons for niche adaptation in Sinorhizobium meliloti. Nat Commun. 2016;7:12219.

21. Lundberg DS, Lebeis SL, Paredes SH, Yourstone S, Gehring J, Malfatti S, et al. Defining the core Arabidopsis thaliana root microbiome. Nature. 2012;488:86-90.

22. Bulgarelli D, Rott M, Schlaeppi K, Ver Loren van Themaat E, Ahmadinejad N, Assenza F, et al. Revealing structure and assembly cues for Arabidopsis root-inhabiting bacterial microbiota. Nature. 
2012;488:91-5.

23. Thiergart T, Duran P, Ellis T, Vannier N, Garrido-Oter R, Kemen E, et al. Root microbiota assembly and adaptive differentiation among European Arabidopsis populations. Nat Ecol Evol. 2020;4:122-31.

24. Hartman K, van der Heijden MGA, Wittwer RA, Banerjee S, Walser JC, Schlaeppi K. Cropping practices manipulate abundance patterns of root and soil microbiome members paving the way to smart farming. Microbiome. 2018;6:14.

25. Schmidt JE, Kent AD, Brisson VL, Gaudin ACM. Agricultural management and plant selection interactively affect rhizosphere microbial community structure and nitrogen cycling. Microbiome. 2019;7:146.

26. Cregger MA, Veach AM, Yang ZK, Crouch MJ, Vilgalys R, Tuskan GA, et al. The Populus holobiont: dissecting the effects of plant niches and genotype on the microbiome. Microbiome. 2018;6:31.

27. Beckers B, Op De Beeck M, Weyens N, Boerjan W, Vangronsveld J. Structural variability and niche differentiation in the rhizosphere and endosphere bacterial microbiome of field-grown poplar trees. Microbiome. 2017;5:25.

28. Edwards J, Johnson C, Santos-Medellín C, Lurie E, Podishetty NK, Bhatnagar S, et al. Structure, variation, and assembly of the root-associated microbiomes of rice. Proc Natl Acad Sci U S A. 2015;112:E911-E20.

29. Xiong C, Zhu YG, Wang JT, Singh BK, Han LL, Shen JP, et al. Host selection shapes crop microbiome assembly and network complexity. New Phytologist. 2021;229:1091-104.

30. Lindow SE, Brandl MT. Microbiology of the phyllosphere. Appl Environ Microbiol. 2003;69:1875-83.

31. Gao C, Montoya L, Xu L, Madera M, Hollingsworth J, Purdom E, et al. Fungal community assembly in drought-stressed sorghum shows stochasticity, selection, and universal ecological dynamics. Nat Commun. 2020;11:1-14.

32. Grady KL, Sorensen JW, Stopnisek N, Guittar J, Shade A. Assembly and seasonality of core phyllosphere microbiota on perennial biofuel crops. Nat Commun. 2019;10:4135.

33. Zhang JY, Zhang N, Liu YX, Zhang XN, Hu B, Qin Y, et al. Root microbiota shift in rice correlates with resident time in the field and developmental stage. Science China Life Sciences. 2018;61:613-21.

34. Chen S, Waghmode TR, Sun R, Kuramae EE, Hu C, Liu B. Root-associated microbiomes of wheat under the combined effect of plant development and nitrogen fertilization. Microbiome. 2019;7:136.

35. Zhao ML, Zhao J, Yuan J, Hale L, Wen T, Huang QW, et al. Root exudates drive soil-microbe-nutrient feedbacks in response to plant growth. Plant Cell Environ. 2021;44:613-28.

36. Agler MT, Ruhe J, Kroll S, Morhenn C, Kim ST, Weigel D, et al. Microbial hub taxa link host and abiotic factors to plant microbiome variation. PLoS Biol. 2016;14:e1002352.

37. van der Heijden MGA, Hartmann M. Networking in the plant microbiome. PLoS Biol. 2016;14:e1002378.

38. Barberan A, Bates ST, Casamayor EO, Fierer N. Using network analysis to explore co-occurrence patterns in soil microbial communities. ISME J. 2012;6:343-51. 
39. Toju $\mathrm{H}$, Tanabe AS, Sato $\mathrm{H}$. Network hubs in root-associated fungal metacommunities. Microbiome. 2018;6:116.

40. Coyte KZ, Schluter J, Foster KR. The ecology of the microbiome: networks, competition, and stability. Science. 2015;350:663-6.

41. Duran $P$, Thiergart $T$, Garrido-Oter $R$, Agler $M$, Kemen $E$, Schulze-Lefert $P$, et al. Microbial interkingdom interactions in roots promote Arabidopsis survival. Cell. 2018;175:973-83.e14.

42. Xiong C, He JZ, Singh BK, Zhu YG, Wang JT, Li PP, et al. Rare taxa maintain the stability of crop mycobiomes and ecosystem functions. Environ Microbiol. 2021;doi: 10.1111/1462-2920.15262.

43. Marx MC, Wood M, Jarvis SC. A microplate fluorimetric assay for the study of enzyme diversity in soils. Soil Biol Biochem. 2001;33:1633-40.

44. Tabatabai M. Soil enzymes. In: R.W. Weaver SA, P. Bottomley, D. Bezdicek, S. Smith, A. Tabatabai and A. Wollum, editors. Methods of soil analysis. Madison: Soil Science Society of America; 1994. p. 775833.

45. Kembel SW, O'Connor TK, Arnold HK, Hubbell SP, Wright SJ, Green JL. Relationships between phyllosphere bacterial communities and plant functional traits in a neotropical forest. Proc Natl Acad Sci U S A. 2014;111:13715-20.

46. Ihrmark K, Bodeker IT, Cruz-Martinez K, Friberg H, Kubartova A, Schenck J, et al. New primers to amplify the fungal ITS2 region-evaluation by 454-sequencing of artificial and natural communities. FEMS Microbiol Ecol. 2012;82:666-77.

47. White TJ, Bruns T, Lee S, Taylor J. Amplification and direct sequencing of fungal ribosomal RNA genes for phylogenetics. In: Innis MA, Gelfand DH, Sninsky JJ, White TJ, editors. PCR protocols. San Diego: Academic Press; 1990. p. 315-22.

48. Edgar RC. Search and clustering orders of magnitude faster than BLAST. Bioinformatics. 2010;26:2460-1.

49. Edgar RC. UNOISE2: improved error-correction for Illumina 16S and ITS amplicon sequencing. bioRxiv. 2016:081257.

50. Caporaso JG, Kuczynski J, Stombaugh J, et al. QIIME allows analysis of highthroughput community sequencing data. Nat Methods. 2010;7:335-6.

51. Paulson JN, Stine OC, Bravo HC, Pop M. Differential abundance analysis for microbial marker-gene surveys. Nat Methods. 2013;10:1200-2.

52. Bolger AM, Lohse M, Usadel B. Trimmomatic: a flexible trimmer for Illumina sequence data. Bioinformatics. 2014;30:2114-20.

53. Langmead B, Salzberg SL. Fast gapped-read alignment with Bowtie 2. Nat Methods. 2012;9:357-9.

54. Li D, Liu CM, Luo R, Sadakane K, Lam TW. MEGAHIT: an ultra-fast single-node solution for large and complex metagenomics assembly via succinct de Bruijn graph. Bioinformatics. 2015;31:1674-6.

55. Seemann T. Prokka: rapid prokaryotic genome annotation. Bioinformatics. 2014;30:2068-9. 
56. Huerta-Cepas J, Szklarczyk D, Heller D, Hernández-Plaza A, Forslund SK, Cook H, et al. eggNOG 5.0: a hierarchical, functionally and phylogenetically annotated orthology resource based on 5090 organisms and 2502 viruses. Nucleic Acids Res. 2018;47:D309-D14.

57. Nakagawa S, Schielzeth $\mathrm{H}, \mathrm{O}$ 'Hara RB. A general and simple method for obtaining $\mathrm{r}^{2}$ from generalized linear mixed-effects models. Methods Ecol Evol. 2013;4:133-42.

58. Oksanen J, Kindt R, Legendre P, O'Hara B, Stevens MHH, Oksanen MJ, et al. The vegan package. Community ecology package. 2007;10:631-7.

59. Stegen JC, Lin XJ, Fredrickson JK, Chen XY, Kennedy DW, Murray CJ, et al. Quantifying community assembly processes and identifying features that impose them. ISME J. 2013;7:2069-79.

60. Jiao S, Yang Y, Xu Y, Zhang J, Lu Y. Balance between community assembly processes mediates species coexistence in agricultural soil microbiomes across eastern China. ISME J. 2020;14:202-16.

61. Zhou J, Deng Y, Zhang P, Xue K, Liang Y, Van Nostrand JD, et al. Stochasticity, succession, and environmental perturbations in a fluidic ecosystem. Proc Natl Acad Sci U S A. 2014;111:E836-E45.

62. Faust K, Sathirapongsasuti JF, Izard J, Segata N, Gevers D, Raes J, et al. Microbial co-occurrence relationships in the human microbiome. PLoS Comput Biol. 2012;8:e1002606.

63. Shannon P, Markiel A, Ozier O, Baliga NS, Wang JT, Ramage D, et al. Cytoscape: a software environment for integrated models of biomolecular interaction networks. Genome research. 2003;13:2498-504.

64. Bastian M, Heymann S, Jacomy M. Gephi: an open source software for exploring and manipulating networks. Icwsm. 2009;8:361-2.

65. Louca S, Parfrey LW, Doebeli M. Decoupling function and taxonomy in the global ocean microbiome. Science. 2016;353:1272-7.

66. Nguyen NH, Song ZW, Bates ST, Branco S, Tedersoo L, Menke J, et al. FUNGuild: an open annotation tool for parsing fungal community datasets by ecological guild. Fungal Ecology. 2016;20:241-8.

67. Knights D, Kuczynski J, Charlson ES, Zaneveld J, Mozer MC, Collman RG, et al. Bayesian communitywide culture-independent microbial source tracking. Nat Methods. 2011;8:761-3.

68. Robinson MD, McCarthy DJ, Smyth GK. edgeR: a Bioconductor package for differential expression analysis of digital gene expression data. Bioinformatics. 2010;26:139-40.

69. Liaw A, Wiener M. Classification and regression by randomForest. R news. 2002;2:18-22.

70. Singh BK, Trivedi P. Microbiome and the future for food and nutrient security. Microb biotechnol. 2017;10:50-3.

71. Chaparro JM, Badri DV, Vivanco JM. Rhizosphere microbiome assemblage is affected by plant development. ISME J. 2013;8:790-803.

72. Huang AC, Jiang T, Liu YX, Bai YC, Reed J, Qu B, et al. A specialized metabolic network selectively modulates Arabidopsis root microbiota. Science. 2019;364:eaau6389.

73. Foster KR, Schluter J, Coyte KZ, Rakoff-Nahoum S. The evolution of the host microbiome as an ecosystem on a leash. Nature. 2017;548:43-51. 
74. Sasse J, Martinoia E, Northen T. Feed your friends: do plant exudates shape the root microbiome? Trends Plant Sci. 2018;23:25-41.

75. Guttman DS, McHardy AC, Schulze-Lefert P. Microbial genome-enabled insights into plantmicroorganism interactions. Nat Rev Genet. 2014;15:797-813.

76. Kudjordjie EN, Sapkota R, Steffensen SK, Fomsgaard IS, Nicolaisen M. Maize synthesized benzoxazinoids affect the host associated microbiome. Microbiome. 2019;7:59.

77. Hu J, Wei Z, Kowalchuk GA, Xu Y, Shen Q, Jousset A. Rhizosphere microbiome functional diversity and pathogen invasion resistance build up during plant development. Environ Microbiol. 2020;22: 5005-18.

78. Vacher C, Hampe A, Porté AJ, Sauer U, Compant S, Morris CE. The phyllosphere: microbial jungle at the plant-climate interface. Annu Rev Ecol Evol S. 2016;47:1-24.

79. Remus-Emsermann MNP, Schlechter RO. Phyllosphere microbiology: at the interface between microbial individuals and the plant host. New Phytologist. 2018;218:1327-33.

80. Vorholt JA. Microbial life in the phyllosphere. Nat Rev Microbiol. 2012;10:828-40.

81. Finkel OM, Salas-Gonzalez I, Castrillo G, Conway JM, Law TF, Teixeira PJPL, et al. A single bacterial genus maintains root growth in a complex microbiome. Nature. 2020;587:103-8.

82. Santolini M, Barabasi AL. Predicting perturbation patterns from the topology of biological networks. Proc Natl Acad Sci U S A. 2018;115:E6375-E83.

83. Banerjee S, Walder F, Buchi L, Meyer M, Held AY, Gattinger A, et al. Agricultural intensification reduces microbial network complexity and the abundance of keystone taxa in roots. ISME J. 2019;13:172236.

84. Alvarez-Perez JM, Gonzalez-Garcia S, Cobos R, Olego MA, Ibanez A, Diez-Galan A, et al. Use of endophytic and rhizosphere Actinobacteria from grapevine plants to reduce nursery fungal graft infections that lead to young grapevine decline. Appl Environ Microbiol. 2017;83:e01564-17.

85. Lee SM, Kong HG, Song GC, Ryu CM. Disruption of Firmicutes and Actinobacteria abundance in tomato rhizosphere causes the incidence of bacterial wilt disease. ISME J. 2021;15:330-47.

86. Viaene T, Langendries S, Beirinckx S, Maes M, Goormachtig S. Streptomyces as a plant's best friend? FEMS Microbiol Ecol. 2016;92:fiw119.

87. Conn VM, Walker AR, Franco CMM. Endophytic actinobacteria induce defense pathways in Arabidopsis thaliana. Molecular Plant-Microbe Interactions. 2008;21:208-18.

88. Trivedi P, Delgado-Baquerizo M, Trivedi C, Hamonts K, Anderson IC, Singh BK. Keystone microbial taxa regulate the invasion of a fungal pathogen in agro-ecosystems. Soil Biol Biochem. 2017;111:104.

89. Hyde KD, Jones EG, Liu JK, Ariyawansa H, Boehm E, Boonmee S, et al. Families of Dothideomycetes. Fungal Diversity. 2013;63:1-313.

90. Adams RI, Miletto M, Taylor JW, Bruns TD. Dispersal in microbes: fungi in indoor air are dominated by outdoor air and show dispersal limitation at short distances. ISME J. 2013;7:1262-73. 
91. Egidi E, Delgado-Baquerizo M, Plett JM, Wang J, Eldridge DJ, Bardgett RD, et al. A few Ascomycota taxa dominate soil fungal communities worldwide. Nat Commun. 2019;10:1-9.

92. Cheng YT, Zhang L, He SY. Plant-microbe interactions facing environmental challenge. Cell Host Microbe. 2019;26:183-92.

93. Toju H, Peay KG, Yamamichi M, Narisawa K, Hiruma K, Naito K, et al. Core microbiomes for sustainable agroecosystems. Nat Plants. 2018;4:247-57.

\section{Figures}
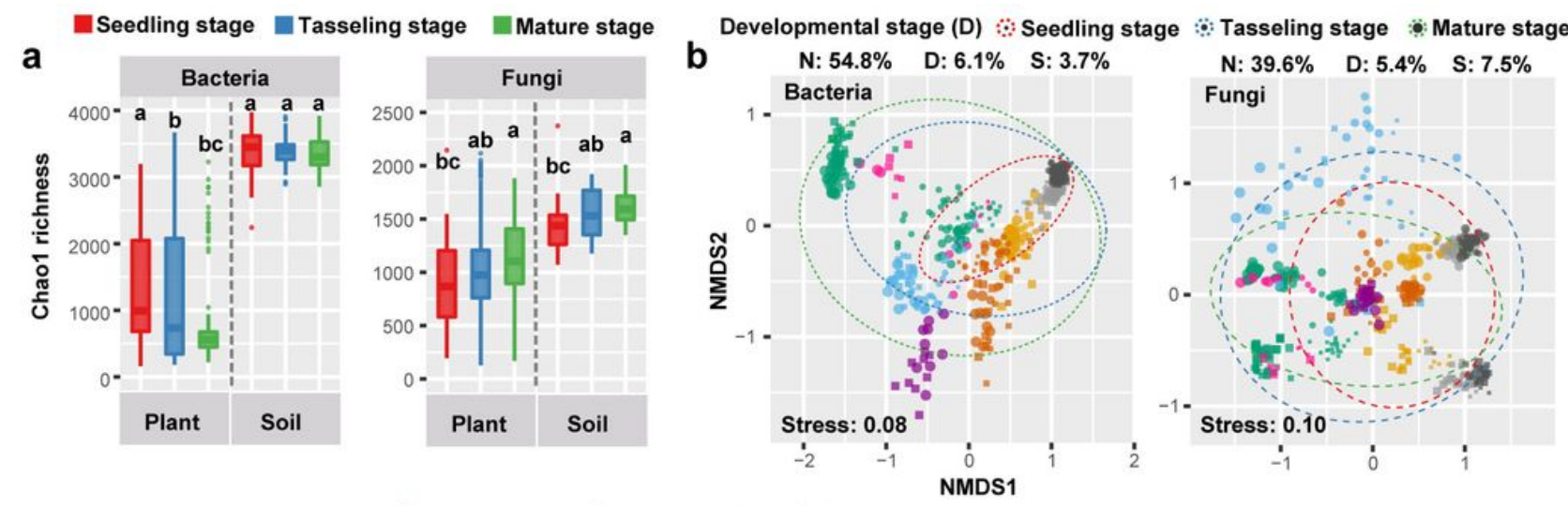

Site (S) •XC $\square$ QJ

Niche (N)

Plastic leaf

Phylloplane

Leaf endosphere

Rhizoplane

Root endosphere

Grain

Rhizosphere soil

Bulk soil
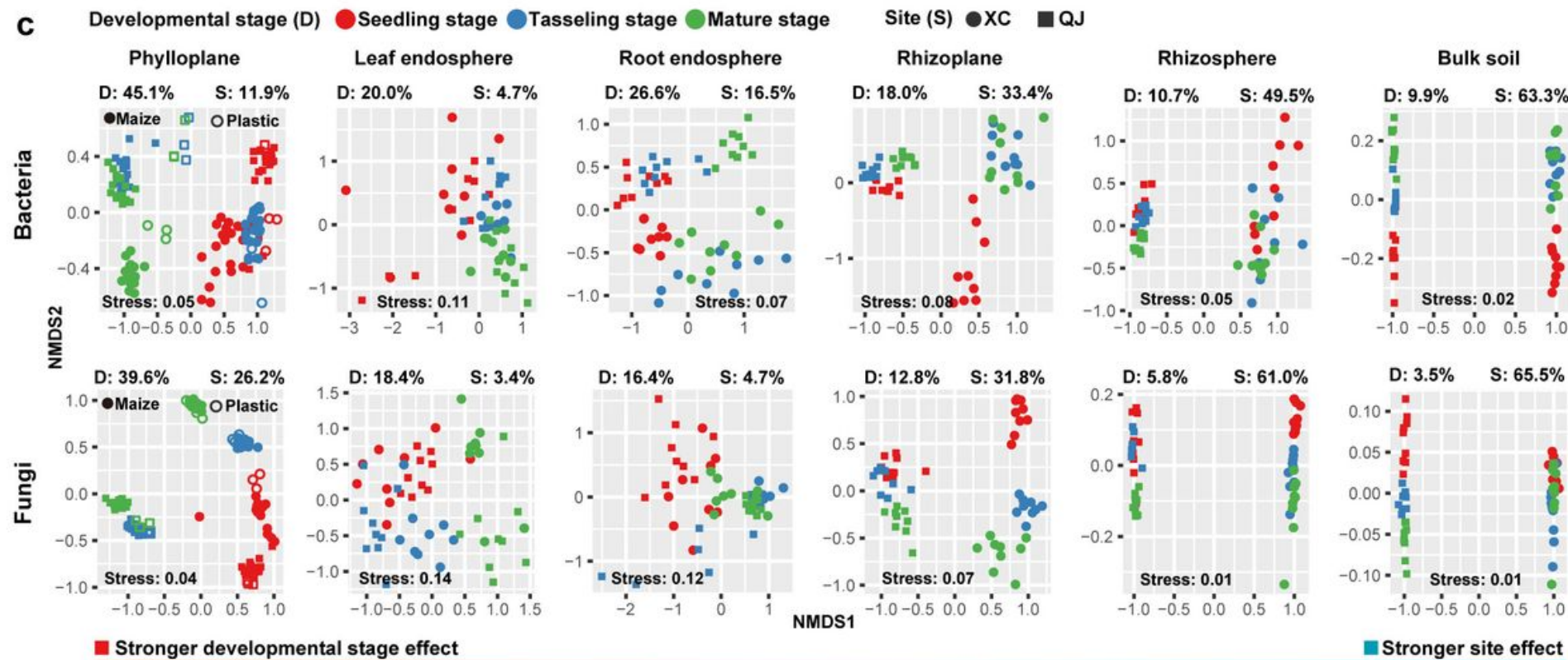

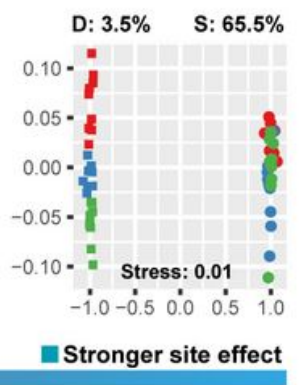

Soil

\section{Figure 1}

Temporal dynamics of diversity and distribution patterns of crop-associated microbiomes. a Alphadiversity of bacterial and fungal communities in plant compartments (phylloplane, leaf endosphere, rhizoplane and root endosphere) and soils (rhizosphere and bulk soil) across three plant developmental stages. b Nonmetric multidimensional scaling (NMDS) ordinations based on weighted UniFrac distances matrices depicting the distribution patterns of bacterial and fungal communities along the soil-plant 
continuum ( $n=432)$. Different letters above the boxes indicate a significant difference determined by nonparametric Kruskal Wallis test. The relative contribution of different factors on community dissimilarity was tested with PERMANOVA. "N" represents the effect of compartment niche, " $D$ " represents the effect of developmental stage, "S" represents the effect of site. "XC" represents site "Xuchang, "QJ" represents site "Qujing. c NMDS ordinations based on weighted UniFrac distances matrices of bacterial and fungal communities in each compartment niche (phylloplane: $n=144$; other niches: $\mathrm{n}=54$ ).

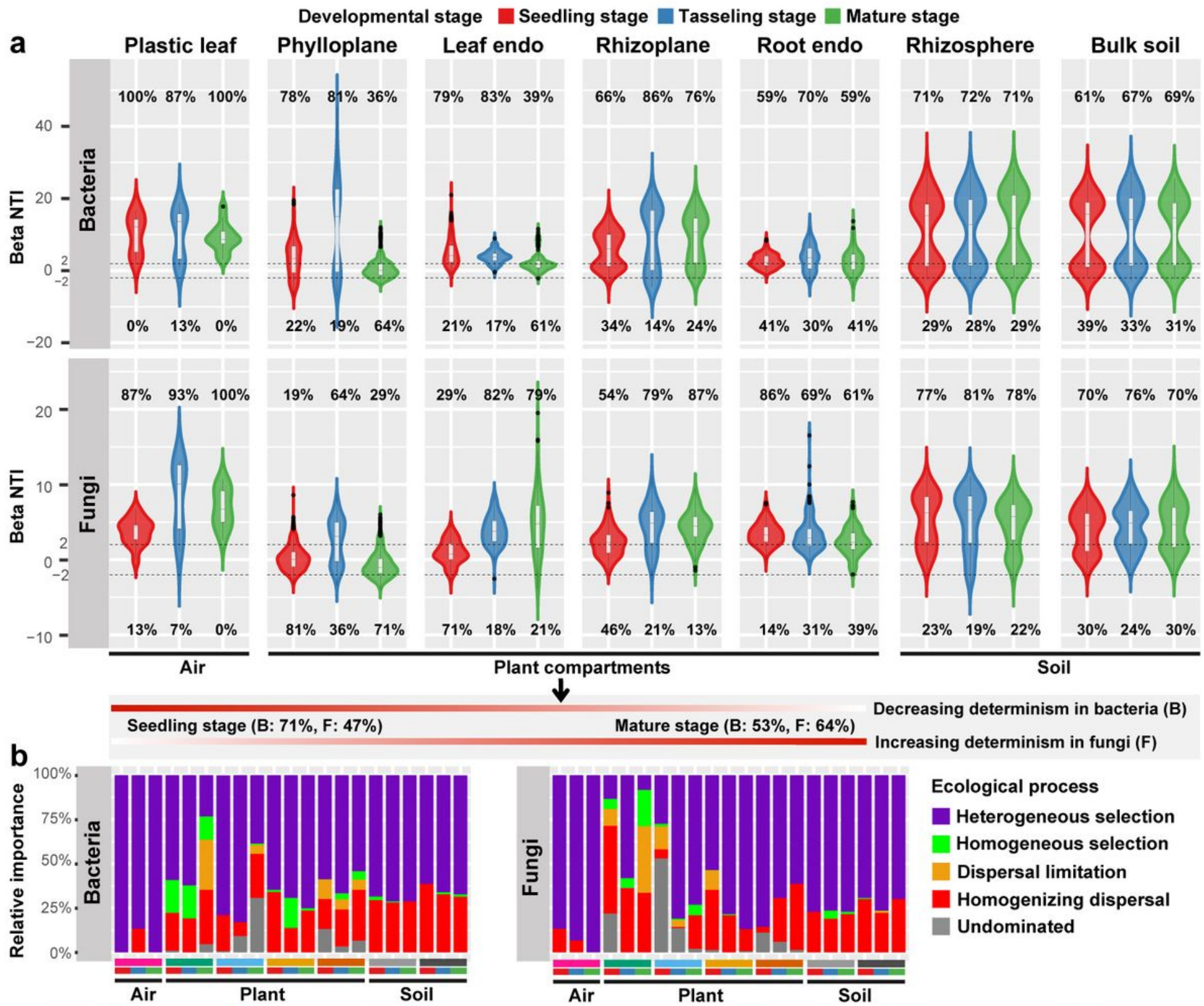

Niche Plastic leaf Leaf endosphere Root endosphere OBulk soil Stage —Seedling stage $\square$ Tasseling stage $\square$ Mature stage Phylloplane Rhizoplane Rhizosphere soil

\section{Figure 2}

Deterministic and stochastic processes in microbiome assembly. a Relative contribution of determinism and stochasticity on microbiome assembly along the soil-plant continuum based on the $\beta$-Nearest Taxon Index ( $\beta N T I$ ) values. The $\beta N T I$ values were calculated using null model, and $\mid \beta N T \| \geq 2$ and $\mid \beta N T \|<2$ 
represents dominant determinism and stochasticity in driving microbiome assembly, respectively. The percentage above and below the violin plot represents the proportion of the deterministic processes and stochastic processes in microbiome assembly, respectively. $b$ The relative importance of five ecological processes (heterogeneous selection: $\beta N T I<-2$, homogeneous selection: $\beta N T I>+2$, dispersal limitation: | $\beta N T||<2$ and RCBray $>0.95$, homogenizing dispersal: $|\beta N T| \mid<2$ and RCBray $<-0.95$, and undominated: $\mid$ $\beta N T I \mid<2$ and $|R C B r a y|<0.95)$ along the soil-plant continuum based on the $\beta$-Nearest Taxon Index ( $\beta N T I)$ and Bray-Curtis-based Raup-Crick Index (RCBray).

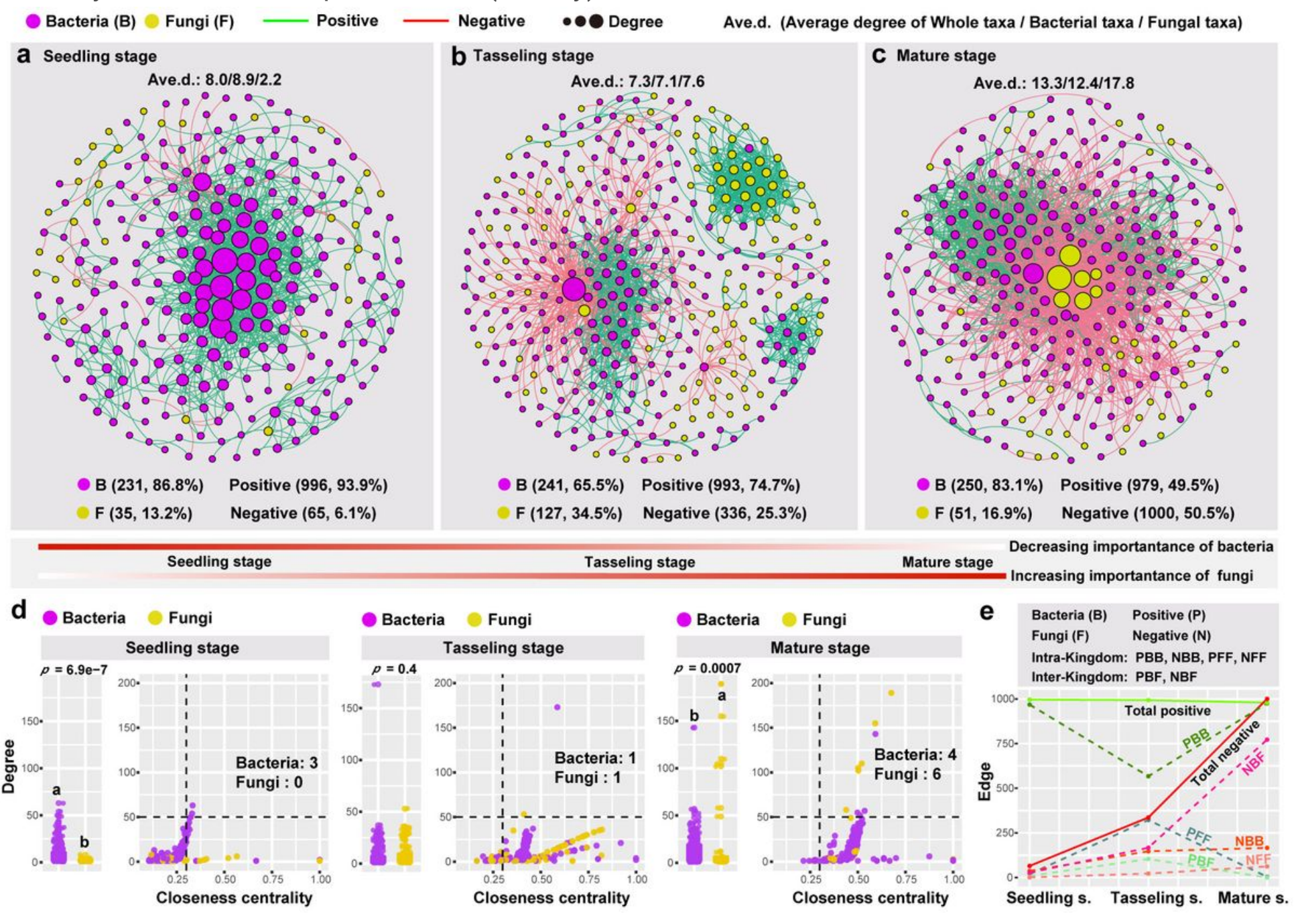

\section{Figure 3}

Temporal dynamics of bacterial-fungal interkingdom networks. Co-occurrence network analysis of full dataset (except for maize grain sample, $n=414$ ) showing microbial interkingdom network patterns differed among a) seedling stage, b) tasseling stage, and c) mature stage. $d$ Comparison of node-level topological features (degree and closeness centrality) between bacterial and fungal taxa at different developmental stages. Different letters indicate a significant difference determined by nonparametric Wilcoxon rank test. e Multiple correlations between bacterial and fungal taxa in interkingdom networks at different developmental stages. 

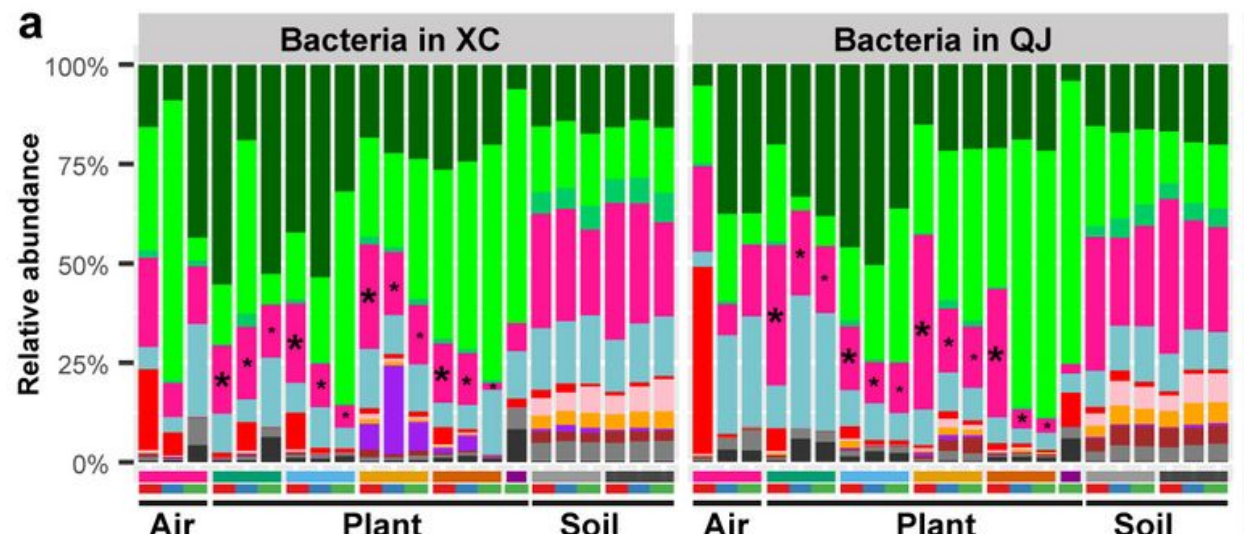

Bacteria
Alphaproteobacteria
Gammaproteobacteria
Deltaproteobacteria
Actinobacteria
Bacteroidetes
Firmicutes
Gemmatimonadetes
Acidobacteria
Patescibacteria
Chloroflexi
Other
Unclassified

Niche

-Plastic leaf

Phylloplane

Leaf endosphere

Rhizoplane

Root endosphere

Grain

Rhizosphere soil

-Bulk soil

Developmental stage

needling stage

- Tasseling stage

Mature stage
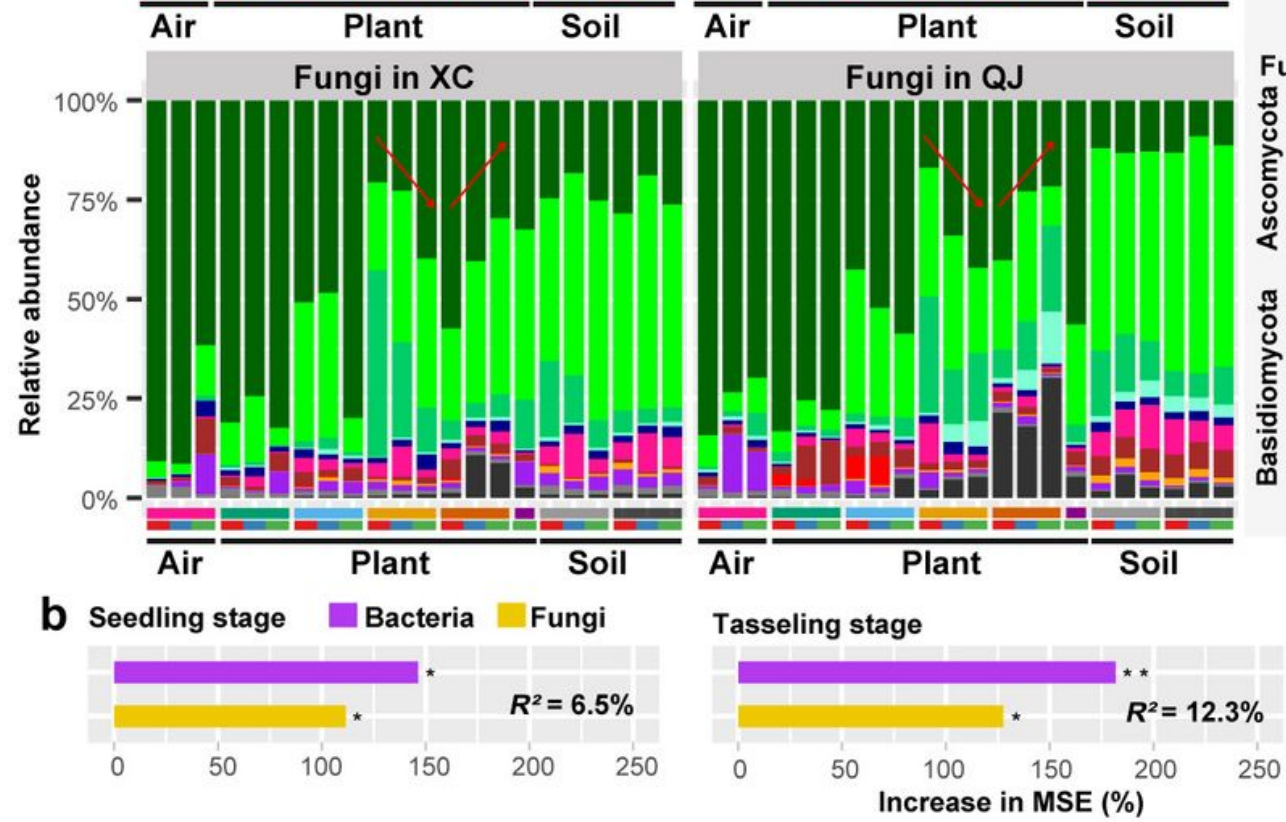

Fungi

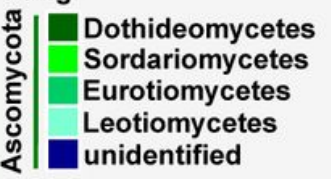

Mortierellomycota.

Mortierellomycetes Other

Fungi.unidentified

Unclassified

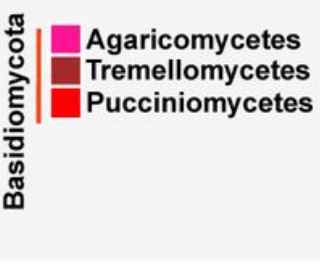

Mature stage

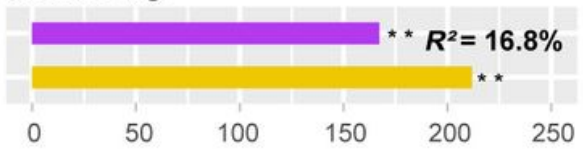

Figure 4

Taxonomic composition and ecological importance of bacterial and fungal microbiomes. a Temporal dynamics of both bacterial and fungal community composition at two study sites. "XC" represents site "Xuchang, "QJ" represents site "Qujing. Low abundance phyla/classes with less than $1 \%$ of the total sequences across all samples are grouped into "Other". b Results from random forest modeling analyses aiming to identify the importance of community composition of bacterial and fungal taxa in predicting crop yield. ${ }^{*} \mathrm{P}<0.05,{ }^{\star} \mathrm{P}<0.001$. Increase in the percentage of MSE is equal to the increase in the mean square error. 


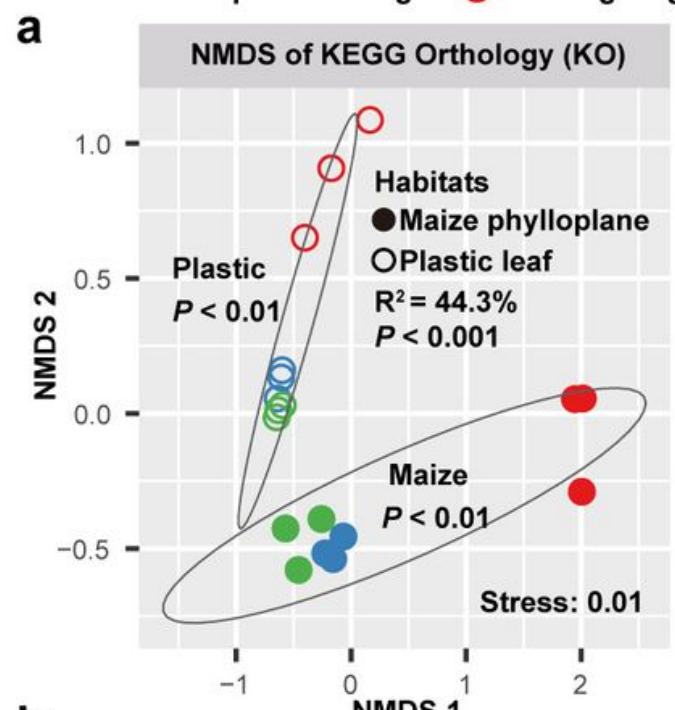

b

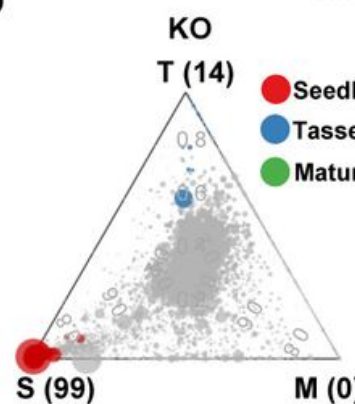

S: K13457, K09422,

$\mathrm{K} 10727$, etc.

K13457 (RPM1, disease resistance protein )

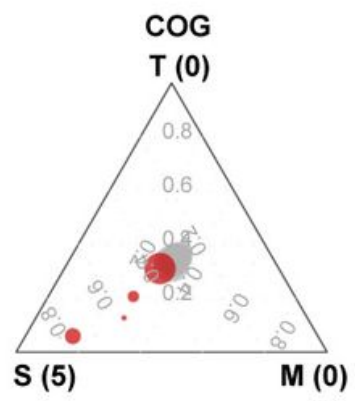

S: A, B, L, W, Z CAZy ing stage (S) T (0)
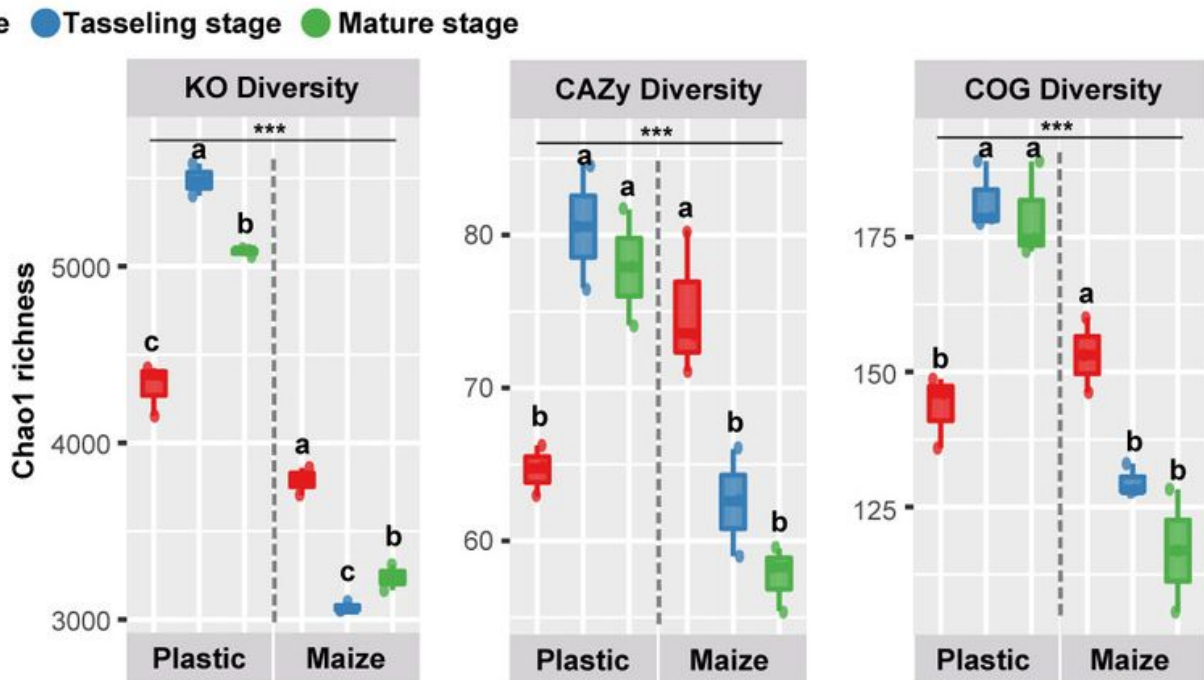

C C fixation N cycling
Tasseli stage Mature stage Denitrification $\square$ Phosphatase

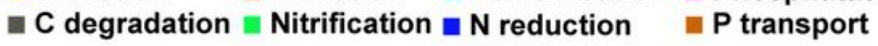

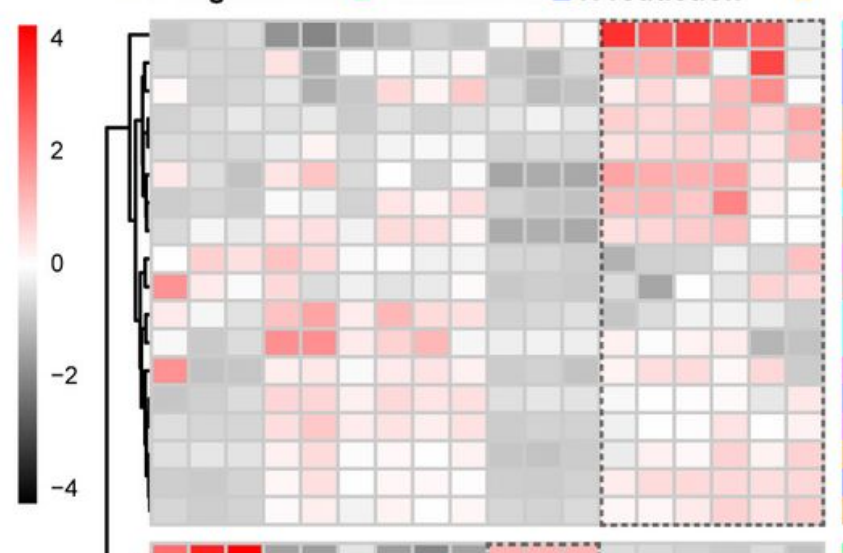

K00376 nosZ K00360 nasB K00372 nasA K01805 xyIA K02040 pstS K02038 pstA K00363 nirD K01176 amyA K01113 phoD K03862 vanA K00368 nirk K02305 norC K09474 phoN K00366 nirA K02039 phoU K02036 pstB K00362 nirB K02037 pstC GH (Glycoside Hydrolases ) GT (Glycosyl Transferases )

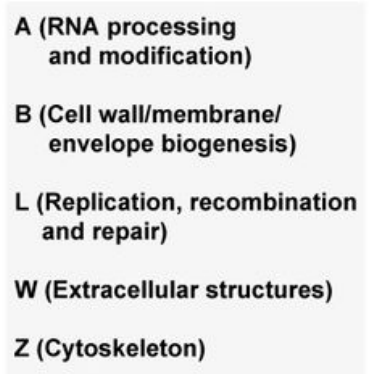

4

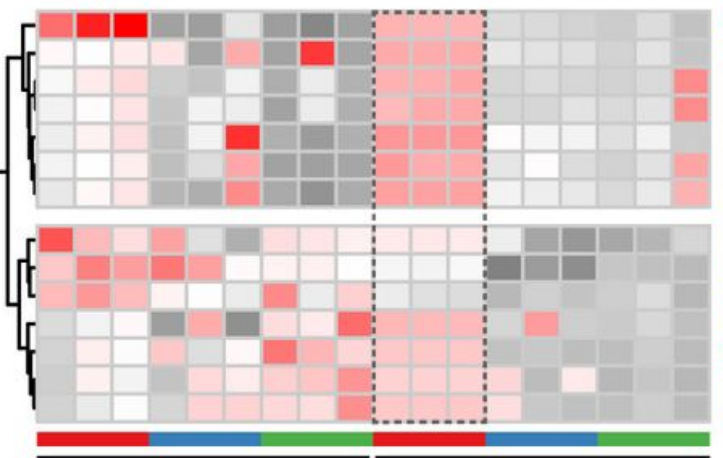

K10944 amoA K03788 aphA K00370 narG K00371 narH K03385 nrfA K02567 napA K02568 napB

K04561 norB K01078 pho K01601 cbbL K02586 nifD K15864 nirS K02588 nifH K02591 nifK

Plastic leaf

\section{Figure 5}

Functional profiles of phylloplane microbiomes. a Nonmetric multidimensional scaling (NMDS) ordinations based on Bray-Curtis distances matrices of KEGG Orthology (KO) showing the maize phylloplane microbiome significantly differed from the plastic leaf microbiome $(n=18)$, and the boxplot showing the functional diversity (include KO, CAZy and COG) of plastic leaf and maize phylloplane microbiomes across three developmental stages. The relative contribution of different factors on microbiome functional composition was tested with PERMANOVA. Different letters above the boxes indicate a significant difference determined by nonparametric Kruskal Wallis test. b Ternary plots 
depicting functional profiles (include KO, CAZy and $\mathrm{COG}$ ) of maize phylloplane microbiome significantly enriched at the seedling stage (FDR, $P<0.01)$. Each circle represents one ZOTU, and the size of each circle represents its relative abundance. $c$ Heat map exhibiting the relative abundance (Z-score) of functional genes (based on $\mathrm{KO}$ ) involved in $\mathrm{C}, \mathrm{N}, \mathrm{P}$ cycling varied among three developmental stages.

\section{Supplementary Files}

This is a list of supplementary files associated with this preprint. Click to download.

- Supplementarylnformation.docx 